\title{
Estimation of Multiple Faults in Aircraft Gas-turbine Engines ${ }^{\star}$
}

\author{
Soumik Sarkar Chinmay Rao Asok Ray \\ szs200epsu.edu crr164epsu.edu axr2epsu.edu \\ Department of Mechanical Engineering \\ The Pennsylvania State University \\ University Park, PA 16802, USA \\ Keywords: Aircraft Propulsion; Gas Turbine Engines; Multiple fault estimation; Statistical Pattern Recognition
}

\begin{abstract}
This paper presents estimation of multiple faults in aircraft gas-turbine engines, based on a statistical pattern recognition tool called Symbolic Dynamic Filtering $(S D F)$. The underlying concept is built upon statistical analysis of evidences to estimate anomalies in multiple critical parameters of the engine system; it also presents a framework for sensor information fusion. The fault estimation algorithm is validated by numerical simulation on the NASA C-MAPSS test-bed of commercial aircraft engines.
\end{abstract}

\section{INTRODUCTION}

Aircraft propulsion system health monitoring is one of the key issues regarding aviation safety. Current state of the art of health monitoring involves a regular and fixed schedule of inspection and maintenance which are essentially conservative in nature and hence may not be cost effective. On the other hand, in unusual circumstances, the normal ground inspection schedule may not be able to detect aggravation of hidden faults, which may result in a permanent damage of the engine or a potentially catastrophic accident. Hence, there is a need for new technologies of health monitoring for aircraft gas turbine engines, which can be pursued either onboard during a flight or on the ground but without the need for installation of additional sensors and/or mounting the engine on a maintenance test facility. Hoffman and van der Merwe [1] have shown that traditional frequencybased methods may not be readily applicable to estimate the evolution of multiple faults in gas turbine engines; fault dictionaries have been used to alleviate this problem, but they are often infeasible to store in onboard applications because of their large sizes [2].

In a two-part paper [3][4], we have reported an $S D F$ based algorithm for detection and isolation (FDI) of faults (Specifically, faults that cause efficiency degradation in engine components) in aircraft engine subsystems. The major contributions of this paper beyond our previous work in the field of engine health monitoring are listed below.

$\star$ This work has been supported in part by NASA under Cooperative Agreement No. NNX07AK49A and by the U.S. Army Research Office under Grant No. W911NF-07-1-0376. Any opinions, findings and conclusions or recommendations expressed in this publication are those of the authors and do not necessarily reflect the views of the sponsoring agencies.
- Estimation of (simultaneously occurring) multiple faults in aircraft gas-turbine engines.

- Formulation of a framework for sensor data fusion as needed for estimation of multiple faults.

- Data compression as pattern vectors of low-dimension for feature-level sensor fusion as needed for onboard vehicle health monitoring and resilient control.

\section{Multiple Fault Estimation Methodology}

This section describes a statistical methodology for multiple fault estimation in aircraft gas-turbine engines along with a sensor fusion framework.

\section{A. Problem Statement}

Let $\mathcal{S}$ denote a collection of (finitely many) data points in the $n$-dimensional parameter space, where the positive integer $n$ is the number of parameters that are to be estimated. That is, $\mathcal{S}=\left\{\underline{s}^{0}, \underline{s}^{1}, \cdots, \underline{s}^{|\mathcal{S}|-1}\right\}$, on which the training process is executed. In the context of gas-turbine engines, $\underline{s}^{k}$ signifies a particular faulty condition in the set of fault conditions $\mathcal{S}$ under consideration. Let $\underline{s}^{0}$ denote the nominal condition of the engine, and $\mathcal{Y}$ be the set of sensors for the engine system consisting of sensors $y_{j}$ for $j=1,2, \ldots,|\mathcal{Y}|$. Let $\Omega$ be the convex hull of $\mathcal{S}$, which represents the range over which the parameters take values. It is noted that $\Omega$ is a convex and compact subset of the separable space $\mathbb{R}^{n}$. The problem at hand is to statistically estimate fault condition $\underline{s} \in \Omega$, given an experimental data set $\Upsilon$, i.e., to identify the conditional probability density $f(\underline{s} \mid \Upsilon)$. It is noted that $\underline{s} \in \Omega$ may not be one of the points in set $\mathcal{S}$.

The multiple-fault estimation procedure is divided into two steps, which are: (i) Forward Problem/Training, and (ii) Inverse Problem/Testing, as described below in detail.

\section{B. Forward Problem/Training}

In the forward problem, a database of patterns is created at parameter values, $\underline{s}^{k}, \forall k=0,1, \cdots,(|\mathcal{S}|-1)$, by collecting time-series data from sensors $y_{j} \in \mathcal{Y}$. Generation of statistical patterns from time series data is posed as a two-scale problem [5][3]. The fast scale is related to the response time of the process dynamics, over the span of which the process is assumed to be quasi-stationary. The slow 
scale is related to the time span over which deviations (e.g., parametric or non-parametric changes) may occur and exhibit non-stationary dynamics. In the present context, time-series data are collected with the system being quasi-stationary at a particular slow-scale epoch $\underline{s}^{k}$. The procedural steps of the forward problem are presented below.

Time series data acquisition on the fast scale from the available sensors: Time series data sets from each sensor $y_{j} \in \mathcal{Y}$ are collected for each epoch $\underline{s}^{k} \in \mathcal{S}$.

Wavelet/Hilbert transform pre-processing of the timeseries data: The wavelet or Hilbert transforms largely alleviate the difficulties of phase-space partitioning and are particularly effective with noisy data from high-dimensional dynamical systems [3][6].

Maximum Entropy Partitioning of the transformed space at the reference condition of epoch $\underline{s}^{0}$ : This step enables transformation of the pre-processed time series data from the continuous domain to the symbol domain [5] by partitioning the transformed phase space, where the data set from each sensor $y_{j}, j=1, \cdots,|\mathcal{Y}|$, has its own alphabet; for each sensor, a specific symbol is assigned to each partition segment from the respective alphabet. Maximum entropy partitioning [3][6] is constructed separately for different sensor data sets at epoch $\underline{s}^{0}$. These partitions are kept invariant for analysis at subsequent epochs $\underline{s}^{1}, \underline{s}^{2}, \ldots, \underline{s}^{|\mathcal{S}|-1}$ of respective sensor data.

Construction of a probabilistic finite state automaton (PFSA) at the reference condition $\underline{s}^{0}$ and Computation of state probabilities: PFSA are constructed for every sensor data at epoch $\underline{s}^{0}$ and their structures remain invariant for subsequent epochs of each sensor data. Let the $N_{j}$, be the number of states in the PFSA corresponding to the sensor $y_{j}, j=1, \cdots,|\mathcal{Y}|$. The sum of the probabilities of all states is equal to unity, i.e., $\sum_{i=1}^{N_{j}} p_{i}^{j}\left(\underline{s}^{k}\right)=1 \forall j \in$ $\{1, \cdots,|\mathcal{Y}|\} \forall k \in\{0, \cdots,|\mathcal{S}|-1\}$, where $p_{i}^{j}\left(\underline{s}^{k}\right)$ denotes the probability of the $i^{t h}$ state of the PFSA constructed fron time series of $j^{t h}$ sensor at epoch $\underline{s}^{k}$; at most $N_{j}-1$, out of the $N_{j}$ elements of the state probability vector can be independent. Therefore, the pattern for each sensor labeled by $j=1, \cdots,|\mathcal{Y}|$ is represented by a $\left(N_{j}-1\right)$-dimensional row vector $\mathbf{p}^{j} \triangleq\left[p_{1}^{j} \cdots p_{N_{j}-1}^{j}\right] \forall j \in\{1, \cdots,|\mathcal{Y}|\}$; this notation holds for all epochs $\underline{s}^{k} \forall k \in\{0, \cdots,|\mathcal{S}|-1\}$.

Construction of the pattern database: A reference pattern array $\mathcal{P}\left(\underline{s}^{0}\right)$ is constructed by vertical stacking of the reference row vectors, $\mathbf{p}^{j}\left(\underline{s}^{0}\right), j \in\{1, \cdots,|\mathcal{Y}|\}$, as shown below.

$$
\mathcal{P}\left(\underline{s}^{0}\right) \triangleq\left\{\begin{array}{l}
\mathbf{p}^{1}\left(\underline{s}^{0}\right) \\
\mathbf{p}^{2}\left(\underline{s}^{0}\right) \\
\cdots \\
\cdots \\
\mathbf{p}^{|\mathcal{Y}|}\left(\underline{s}^{0}\right)
\end{array}\right\}=\left\{\begin{array}{l}
p_{1}^{1}\left(\underline{s}^{0}\right) \cdots p_{N_{1}-1}^{1}\left(\underline{s}^{0}\right) \\
p_{1}^{2}\left(\underline{s}^{0}\right) \cdots p_{N_{2}-1}^{2}\left(\underline{s}^{0}\right) \\
\cdots \\
\cdots \\
p_{1}^{|\mathcal{Y}|}\left(\underline{s}^{0}\right) \cdots p_{N_{|\mathcal{Y}|}-1}^{|\mathcal{Y}|}\left(\underline{s}^{0}\right)
\end{array}\right\}
$$

Computation of pattern statistics: Different units of identically manufactured engines are different in behavior or performance; this inevitable uncertainty is modeled as the process noise. Therefore, several runs are performed for each fault condition, with a certain value of process noise along with an a priori determined sensor noise (e.g., calculated from instrumentation manufacturer's specifications) to obtain the pattern vector statistics. Let the pattern array $\mathcal{P}\left(\underline{s}^{k}\right)$ be modeled as a random array $\mathcal{Q}\left(\underline{s}^{k}\right)$, whose elements are $q_{i}^{l}\left(\underline{s}^{k}\right)$ that is constructed from the ensemble of realizations $p_{i}^{l}\left(\underline{s}^{k}\right)$. Considering up to second order statistics, elements of the random array $\mathcal{Q}\left(\underline{s}^{k}\right)$ are modeled to have multivariate structures from the perspectives of state machine construction in the $S D F$ setting. Thus, for each epoch $\underline{s}^{k}$, a mean pattern vector $\mu\left(\underline{s}^{k}\right)$ and a corresponding covariance matrix $\Gamma\left(\underline{s}^{k}\right)$ of the pattern are calculated from the elements of $\mathcal{Q}\left(\underline{s}^{k}\right)$. An element of $\mu\left(\underline{s}^{k}\right)$ is expressed as $m_{i}^{l}\left(\underline{s}^{k}\right), \forall l \in$ $\{1,2, \cdots,|\mathcal{Y}|\}$ and $\forall i \in\left\{1,2, \ldots, N_{l}-1\right\}$, which signifies the mean values of $p_{i}^{l}\left(\underline{s}^{k}\right)$ generated from the data sets of different runs. Similarly, an element of the covariance matrix $\Gamma\left(\underline{s}^{k}\right)$ is expressed as $\gamma_{i j}^{l \ell}\left(\underline{s}^{k}\right), \forall l, \ell \in\{1,2, . .|\mathcal{Y}|\}$ and $\forall i \in\left\{1,2, \ldots, N_{l}-1\right\}$, and $\forall j \in\left\{1,2, \ldots, N_{\ell}-1\right\}$, which signifies the value of cross-covariance between $p_{i}^{l}\left(\underline{s}^{k}\right)$ and $p_{j}^{\ell}\left(\underline{s}^{k}\right)$, which is also generated from the data sets of different runs. Note that, for $l=\ell$, the covariance matrix terms yield correlation among the states $i$ and $j$ of the PFSA generated from the same sensor data and, for $l \neq \ell$, the covariance matrix terms yield correlation among the states $i$ and $j$ of different PFSA corresponding to different sensors.

For the purpose of book-keeping in statistical calculations, each of the (two-dimensional) arrays $\mathcal{P}\left(\underline{s}^{k}\right)$ is rearranged as a single row vector $\boldsymbol{p}\left(\underline{s}^{k}\right)$ by horizontally concatenating the row vectors $\mathbf{p}^{j}\left(\underline{s}^{k}\right), j \in\{1, \cdots,|\mathcal{Y}|\}$, i.e., the random pattern array $\mathcal{Q}\left(\underline{s}^{k}\right)$ is rearranged as the random pattern vector $\boldsymbol{q}\left(\underline{s}^{k}\right)$. The mean pattern vector $\mu\left(\underline{s}^{k}\right)$ and covariance matrix $\Gamma\left(\underline{s}^{k}\right)$ are constructed correspondingly. The covariance matrix $\Gamma\left(\underline{s}^{k}\right)$ is comprised of several blocks of elements. The square diagonal blocks correspond to the covariance among states of same sensor data, where as the off-diagonal possibly non-square (due to possible different alphabet size for different sensor data) blocks correspond to the covariance among states of different sensor data.

The (jointly Gaussian as involves only second order statistics) conditional probability distribution of a random pattern vector $\boldsymbol{q}$ is given as

$$
\begin{aligned}
f_{\boldsymbol{q} \mid \Omega}\left(\boldsymbol{p} \mid \underline{s}^{k}\right)= & \frac{1}{(2 \pi)^{N / 2}\left|\Gamma\left(\underline{s}^{k}\right)\right|^{1 / 2}} . \\
& \exp \left(-\frac{1}{2}\left(\boldsymbol{p}-\mu\left(\underline{s}^{k}\right)\right)\left(\Gamma\left(\underline{s}^{k}\right)\right)^{-1}\left(\boldsymbol{p}-\mu\left(\underline{s}^{k}\right)\right)^{T}\right)
\end{aligned}
$$

where $N=N_{1}+\cdots+N_{|\mathcal{Y}|}-|\mathcal{Y}|$. 


\section{Inverse Problem/Testing}

The objective here is to identify the probabilistic location of the fault in the multi-dimensional parameter space, i.e., identification of the unknown parameter vector $\underline{s} \in \Omega$; however, it is possible that $\underline{s} \notin \mathcal{S}$. Therefore, for a particular test case, time series data are collected from different sensors. The data are analyzed using the same symbolic dynamic filter constructed in the forward problem/training (see Section 2$\mathrm{B})$, and the resulting row vector $\boldsymbol{p}$ is a realization of a random pattern vector $\boldsymbol{q}$. The density function $f_{\Omega \mid \boldsymbol{q}}(\underline{s} \mid \boldsymbol{p})$ is obtained as

$$
\begin{aligned}
f_{\Omega \mid \boldsymbol{q}}(\underline{s} \mid \boldsymbol{p}) & =\frac{f_{\boldsymbol{q} \mid \Omega}(\boldsymbol{p} \mid \underline{s}) f_{\Omega}(\underline{s})}{f_{\boldsymbol{q}}(\boldsymbol{p})} \\
& =\frac{f_{\boldsymbol{q} \mid \Omega}(\boldsymbol{p} \mid \underline{s}) f_{\Omega}(\underline{s})}{\int_{\Omega} f_{\boldsymbol{q} \mid \Omega}(\boldsymbol{p} \mid \underline{\tilde{s}}) f_{\Omega}(\underline{\tilde{s}}) d \underline{\tilde{s}}}
\end{aligned}
$$

In the absence of a priori information, an assumption is made that all operating conditions are equally likely, i.e., $f_{\Omega}(\underline{s})=f_{\Omega}(\underline{\tilde{s}}) \forall \underline{\tilde{s}}, \underline{\tilde{s}} \in \Omega$. With this assumption of uniform probability, Eq. (2) reduces to

$$
f_{\Omega \mid \boldsymbol{q}}(\underline{s} \mid \boldsymbol{p})=\frac{f_{\boldsymbol{q} \mid \Omega}(\boldsymbol{p} \mid \underline{s})}{\int_{\Omega} f_{\boldsymbol{q} \mid \Omega}(\boldsymbol{p} \mid \underline{\tilde{s}}) d \underline{\tilde{s}}}
$$

It is noted that accuracy of the above distribution would be improved if the actual prior mapping, i.e., $f_{\Omega}(\underline{s})$ is known. The integral in the denominator of Eq. (3) is approximated by a Reimann sum as

$$
f_{\Omega \mid \boldsymbol{q}}(\underline{s} \mid \boldsymbol{p}) \approx \kappa \frac{f_{\boldsymbol{q} \mid \Omega}(\boldsymbol{p} \mid \underline{s})}{\sum_{\mathcal{S}} f_{\boldsymbol{q} \mid \Omega}(\boldsymbol{p} \mid \underline{\tilde{s}})}
$$

where $\kappa$ is a constant. This approximation converges to the exact solution as the training set $\mathcal{S}$ approaches a countable dense subset of $\Omega \subset \mathbb{R}^{n}$. The density function in Eq. (4) is now sampled at the points $\underline{s}^{k}$ in the training set $\mathcal{S}$ and the following sampled density is constructed as to yield

$$
\left.f_{\Omega \mid \boldsymbol{q}}(\underline{s} \mid \boldsymbol{p})\right|_{\underline{s}=\underline{s}^{k}} \approx \kappa \frac{f_{\boldsymbol{q} \mid \Omega}\left(\boldsymbol{p} \mid \underline{s}^{k}\right)}{\sum_{\underline{\tilde{s}}_{\mathcal{S}}} f_{\boldsymbol{q} \mid \Omega}(\boldsymbol{p} \mid \underline{\tilde{s}})} \forall \underline{s}^{k} \in \mathcal{S}
$$

The density functions in the numerator and denominator of Eq. (5) are obtained from Eq. (1), which were determined in the training phase. It is noted that the nature of the density function $f_{\Omega \mid \boldsymbol{q}}\left(\underline{s}^{k} \mid \boldsymbol{p}\right)$ does not depend on the constant $\kappa$.

The probability mass functions are obtained by evaluating the probability density function in Eq. (5) at points $\underline{s}^{k} \in \mathcal{S}$.

$$
\begin{aligned}
P\left(\underline{s}^{k} \mid \boldsymbol{p}\right) & \triangleq \frac{f_{\Omega \mid \boldsymbol{q}}\left(\underline{s}^{k} \mid \boldsymbol{p}\right)}{\sum_{j=1}^{|\mathcal{S}|} f_{\Omega \mid \boldsymbol{q}}\left(\underline{s}^{j} \mid \boldsymbol{p}\right)} \\
& \approx \frac{f_{\boldsymbol{q} \mid \Omega}\left(\boldsymbol{p} \mid \underline{s}^{k}\right)}{\sum_{j=1}^{|\mathcal{S}|} f_{\boldsymbol{q} \mid \Omega}\left(\boldsymbol{p} \mid \underline{s}^{j}\right)}
\end{aligned}
$$

Following Eq. (1), the above equation is expressed as

$$
\begin{aligned}
P\left(\underline{s}^{k} \mid \boldsymbol{p}\right) \approx \frac{\frac{1}{(2 \pi)^{N / 2}\left|\Gamma\left(\underline{s}^{k}\right)\right|^{1 / 2}}}{\sum_{l=1}^{|\mathcal{S}|} \frac{1}{(2 \pi)^{N / 2}\left|\Gamma\left(\underline{s}^{l}\right)\right|^{1 / 2}}} . \\
\quad \frac{\exp \left(-\frac{1}{2}\left(\boldsymbol{p}-\mu\left(\underline{s}^{k}\right)\right)\left(\Gamma\left(\underline{s}^{k}\right)\right)^{-1}\left(\boldsymbol{p}-\mu\left(\underline{s}^{k}\right)\right)^{T}\right)}{\exp \left(-\frac{1}{2}\left(\boldsymbol{p}-\mu\left(\underline{s}^{l}\right)\right)\left(\Gamma\left(\underline{s}^{l}\right)\right)^{-1}\left(\boldsymbol{p}-\mu\left(\underline{s}^{l}\right)\right)^{T}\right)}
\end{aligned}
$$

The above equation signifies a statistical pattern matching by calculating the Mahalnobis distance [7] between the test and the training patterns; therefore, smaller the Mahalnobis distance, better is the match between these two patterns.

It has been observed from experimental data that fluctuations of the pattern vectors are very weakly correlated among different symbols and different sensors. Therefore, the jointly Gaussian distribution of all $f_{\boldsymbol{q} \mid \Omega}\left(\boldsymbol{p} \mid \underline{s}^{k}\right)$ 's can be reduced to the product of individual Gaussian distributions $f_{\boldsymbol{q} \mid \Omega}\left(p_{i}^{j} \mid \underline{s}^{k}\right)$ of different symbols $\forall j \in\{1,2, . .,|\mathcal{Y}|\}$ and $\forall i \in\left\{1,2, \ldots, N_{j}-1\right\}$. Therefore, instead of using the multivariate jointly Gaussian distribution, univariate Gaussian distribution is used for each symbol, (the variance being the corresponding diagonal element of the covariance matrix) to calculate $P\left(\underline{s}^{k} \mid \boldsymbol{p}\right)$. Thus, Eq. (7) reduces to Eq. (8).

$$
\begin{aligned}
P\left(\underline{s}^{k} \mid \boldsymbol{p}\right) & \approx \frac{\prod_{j} \prod_{i} \frac{1}{(2 \pi)^{1 / 2}\left(\gamma_{i i}^{j j}\left(\underline{s}^{k}\right)\right)^{1 / 2}}}{\sum_{l=1}^{|\mathcal{S}|} \prod_{j} \prod_{i} \frac{1}{(2 \pi)^{1 / 2}\left(\gamma_{i i}^{j j}\left(\underline{s}^{l}\right)\right)^{1 / 2}}} \\
& \frac{\left.\exp \left(-\frac{1}{2}\left(p_{i}^{j}-m_{i}^{j}\left(\underline{s}^{k}\right)\right)\left(\gamma_{i i}^{j j}\left(\underline{s}^{k}\right)\right)^{-1}\left(p_{i}^{j}-m_{i}^{j}\left(\underline{s}^{k}\right)\right)\right)_{(}\right)}{\exp \left(-\frac{1}{2}\left(p_{i}^{j}-m_{i}^{j}\left(\underline{s}^{l}\right)\right)\left(\gamma_{i i}^{j j}\left(\underline{s}^{l}\right)\right)^{-1}\left(p_{i}^{j}-m_{i}^{j}\left(\underline{s}^{l}\right)\right)\right)}
\end{aligned}
$$

$\forall j \in\{1,2, \ldots,|\mathcal{Y}|\}$ and $\forall i \in\left\{1,2, \ldots, N_{j}-1\right\}$.

Once the probability mass function $P\left(\underline{s}^{k} \mid \boldsymbol{p}\right)$ is obtained, there can be different estimates $\underline{\hat{s}} \in \Omega$ depending upon the cost function of estimation. For example, the median of the distribution yields the estimated value by minimizing the root mean square value of the deviations. Again, most likely parameter value can be obtained from the mode of the distribution. In this paper, estimated mean is considered which minimizes the average of the square of the absolute deviations around the estimated point. Estimated mean $\underline{\hat{s}}$ and estimated covariance matrix $\hat{C}_{\underline{s}}$ of the parameter (column) vector $\underline{s}$ are obtained directly from $P\left(\underline{s}^{k} \mid \boldsymbol{p}\right)$ as

$$
\begin{aligned}
\underline{\hat{s}}(\boldsymbol{p}) & \triangleq \sum_{k=1}^{|\mathcal{S}|} \underline{s}^{k} P\left(\underline{s}^{k} \mid \boldsymbol{p}\right) \\
\hat{C}_{\underline{s}}(\boldsymbol{p}) & \triangleq \sum_{k=1}^{|\mathcal{S}|}\left(\underline{s}^{k}-\underline{\hat{s}}(\boldsymbol{p})\right) P\left(\underline{s}^{k} \mid \boldsymbol{p}\right)\left(\underline{s}^{k}-\underline{\hat{s}}(\boldsymbol{p})\right)^{T}(10)
\end{aligned}
$$

Since the statistical information is available in the form of probability mass functions, the third and higher moments of the parameter vector can be estimated in a similar way; 
however, third and higher moments are redundant because the inherent distribution is assumed to have a Gaussian structure that carries full statistical information in the first two moments.

\section{Discussion on Sensor Fusion}

The above formulation of the inverse problem uses information from all the sensors $y_{j}$ for $j=1,2, \ldots,|\mathcal{Y}|$, i.e., the information from all sensors are fused together to estimate the fault level in the engine test case. However, this fusion technique allows the user to choose the number and the combination of sensors to be used for the multiple fault estimation. Suppose only sensors $s_{1}$ and $s_{3}$ are to be used. Then, elements $m_{i}^{j}\left(\underline{s}^{k}\right), \forall j \in\{1,3\}$ and $\forall i \in\left\{1,2, \ldots, N_{j}-1\right\}$ are selected from $\mu\left(\underline{s}^{k}\right)$ and elements $\gamma_{x y}^{m n}\left(\underline{s}^{k}\right), \forall m, n \in\{1,3\}$ and $\forall x \in\left\{1,2, \ldots, N_{m}-1\right\}, \forall y \in\left\{1,2, \ldots, N_{n}-1\right\}$ are selected from $\Gamma\left(\underline{s}^{k}\right) \forall \underline{s}^{k} \in \mathcal{S}$. It follows from the above example that the elements of the test patterns need to be selected corresponding to the sensors under consideration.

Remark 2.1: The current framework attempts to fuse information from different sensors at feature level as opposed to the frameworks of data level or decision level fusion. The advantages of the present sensor information fusion framework are delineated below.

- Data level fusion techniques often encounter scaling problem while fusing information from sensors of different modality. However, the present technique fuses the probability vector patterns, which does not have any scaling issue.

- Decision level fusion generally provides too coarse diagnosis of faults and also requires in depth understanding of the physical system.

\section{VALIDATION ON THE C-MAPSS TEST-BED}

The C-MAPSS simulation test-bed, developed at NASA, is built upon the model of a commercial-scale two-spool turbofan engine and its control system. The details of the model are available in [8], a brief outline of C-MAPSS is provided here for completeness of the paper. The simulation test-bed of the gas turbine engine system consists of five major rotating components namely, fan $(\mathrm{F})$, low pressure compressor (LPC), high pressure compressor (HPC), high pressure turbine (HPT), and low pressure compressor (LPT). Given the inputs of throttle resolving angle (TRA), altitude $(a)$ and Mach number $(M)$, the interactively controlled component models at the simulation test-bed compute nonlinear dynamics of real-time turbofan engine operation. The entire test-bed code is written on Matlab and Simulink platform.

As indicated earlier, this paper addresses estimation of those faults that cause efficiency degradation in engine components. In the current configuration of the C-MAPSS simulation test-bed, there are 13 health parameter inputs, among which the following are considered. For the engine's five rotating components (i.e., Fan, LPC, HPC, HPT and LPT), the ten health parameters are: (a) fan $\left(\psi_{F}, \zeta_{F}\right)$, (b) low pressure compressure $\left(\psi_{L P C}, \zeta_{L P C}\right)$, (c) high pressure compressor $\left(\psi_{H P C}, \zeta_{H P C}\right)$, (d) high pressure turbine $\left(\psi_{H P T}, \zeta_{H P T}\right)$, and (e) low pressure turbine $\left(\psi_{L P T}, \zeta_{L P T}\right)$. Table I lists the (commercially available) sensors and their locations that have been used for multiple fault estimation in C-MAPSS engine test-bed.

TABLE I

REQUIRED ENGINE SYSTEM SENSORS

\begin{tabular}{c|c}
\hline Sensors & Description \\
\hline$P_{24}$ & LPC exit/ HPC inlet pressure \\
$T_{24}$ & LPC exit/ HPC inlet temperature \\
$P s_{30}$ & HPC exit static pressure \\
$T_{30}$ & HPC exit/ Burner inlet temperature \\
$T_{50}$ & LPT exit temperature \\
\hline
\end{tabular}

\section{A. Results and Discussions}

Time series data have been collected for different sensors under persistent excitation of TRA inputs that have truncated triangular profiles with the mean value of $80^{\circ}$, fluctuations within $\pm 2^{\circ}$ and frequency of $0.056 \mathrm{~Hz}$. The ambient conditions are chosen to be at the sea level (i.e. altitude $a=0.0$, Mach number $M=0.0$ ) when the engine is on the ground for fault monitoring and maintenance by the engineering personnel. The engine simulation is conducted at a frequency of $66.67 \mathrm{~Hz}$ (i.e., inter-sample time of $15 \mathrm{~ms}$ ) and the length of the simulation time window is 150 seconds, which generate 10,000 data points for each training or test case.

An engine component $C$ is considered in nominal condition when both $\psi_{C}$ and $\zeta_{C}$ are equal to 1 . Fault is injected in the component $C$ by simultaneously reducing both $\psi_{C}$ and $\zeta_{C}$ by same amount in the results reported in this paper. Although the algorithm described above, does not have any restriction on the dimension of the parameter space, the result presented here considers simultaneous degradation of two different components. Subsection 3-A.1 describes a fault condition, where Fan and LPC are degraded simultaneously, whereas Subsection 3-A.2 analyzes simultaneous degradation in HPT and LPT . For both training (i.e., forward problem) and testing (i.e., inverse problem), time series data from all sensors, listed in Table I, are generated with $\psi$ and $\zeta$ ranging from 1.0 to 0.96 (i.e., $4 \%$ relative loss in efficiency) in steps of 0.005 for the engine components under consideration. For SDF analysis, the number of states in the PFSA is selected to be 15 for each sensor after pre-processing the time series data by Hilbert transform and pattern vectors are generated for each of the possible fault conditions. Fifty repetitions of each simulation have been conducted to generate pattern vector statistics with injected process and sensor noise. For testing (i.e., inverse problem), fault conditions are chosen within the range of training data such that they do not coincide with the training grid points.. The results of multiple-fault estimation are presented in the following two subsections along with discussions on sensor fusion. 


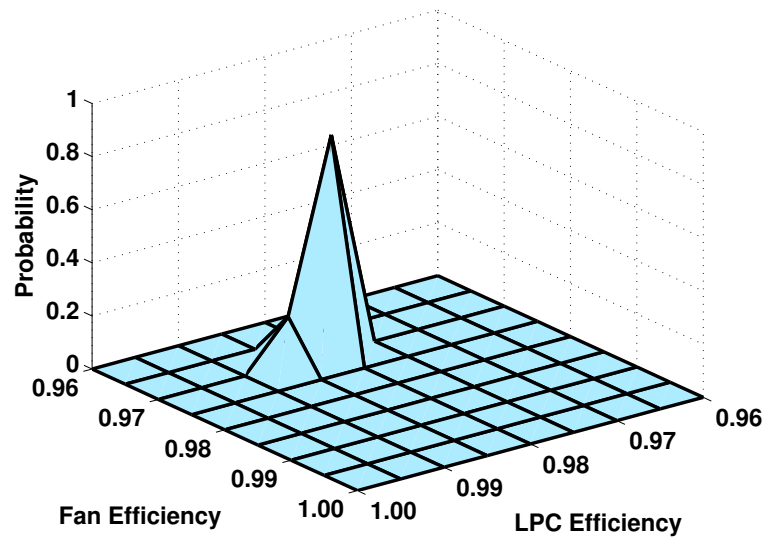

(a) Surface plot of fault estimation

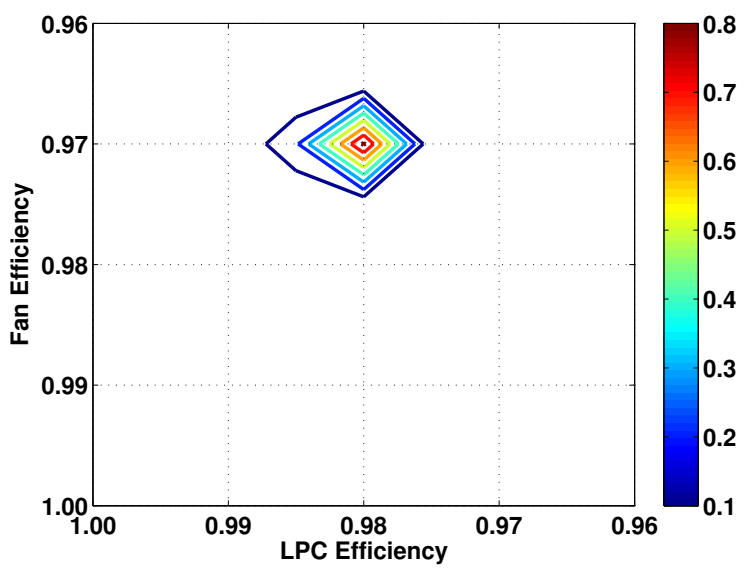

(b) Contour plot of fault estimation

Fig. 1. Fault estimation in Fan-LPC based on $P s_{30}$ sensor

1) Fault estimation in Fan and LPC: A test pattern is generated for a given fault condition, $\psi_{F}=\zeta_{F}=0.973$ and $\psi_{L P C}=\zeta_{L P C}=0.981$. The 3-dimensional plot in Fig. 1(a) shows the bivariate probability distribution of the estimated fault condition, followed by a close-up view of the contour plots in Fig. 1(b), where the results are generated from time series of a single sensor, namely, $P s_{30}$. The estimates lie within the $\pm 3 \sigma$ bound around the estimated mean (see Eq. (9)), where the variance $\sigma^{2}$ is obtained as a diagonal element of the estimated covariance matrix $\hat{C}_{\underline{s}}$ (see Eq. (10)). In this case, the estimates range from 0.9606 to 0.9704 for $\psi_{F}$ and $\zeta_{F}$, and from 0.9805 to 0.9813 for $\psi_{L P C}$ and $\zeta_{L P C}$, respectively. This indicates that the correct region is located in the parameter space, which assigns highest probability to the nearest training grid point.

2) Fault estimation in HPT-LPT: This example shows the result for a fault condition, $\psi_{H P T}=\zeta_{H P T}=0.977$ and $\psi_{L P T}=\zeta_{L P T}=0.985$. In contrast to the previous example of fan and LPC, the plots in Fig. 2(a) and Fig. 2(b) show that there is an ambiguity in estimation when using information from only one sensor, namely $P s_{30}$. Although,

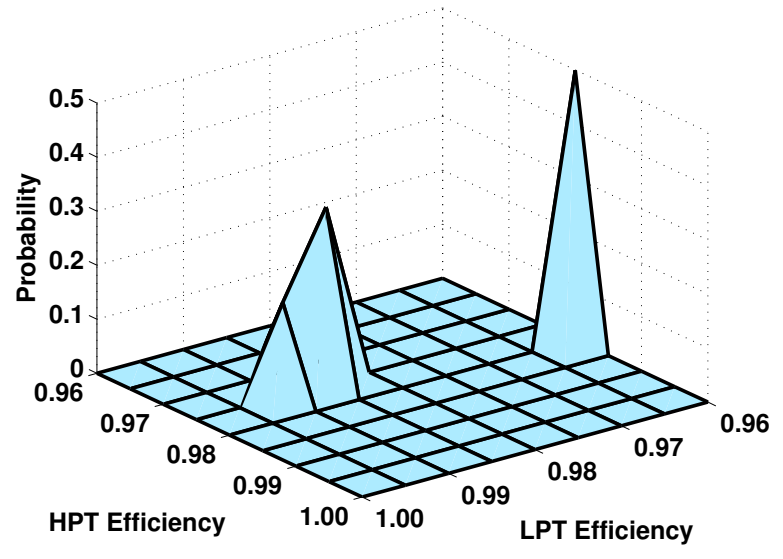

(a) Surface plot of fault estimation

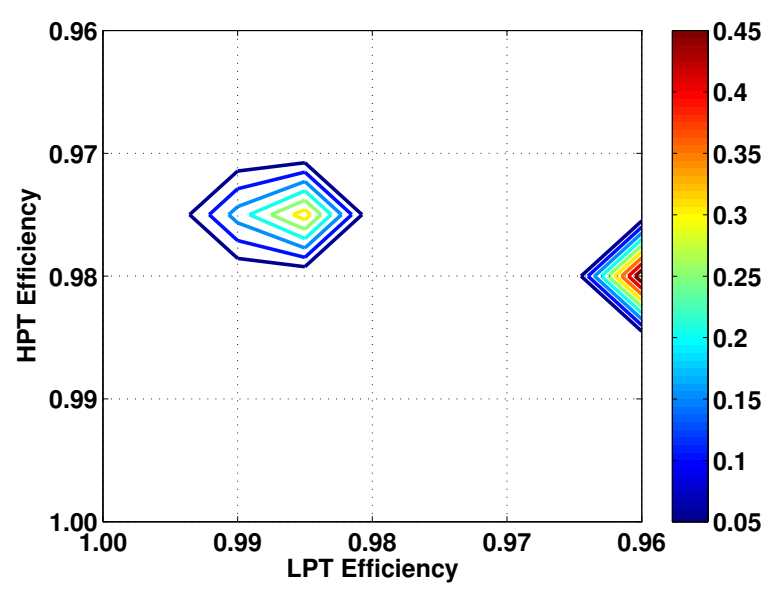

(b) Contour plot of fault estimation

Fig. 2. Fault estimation in HPT-LPT based on $P s_{30}$ sensor

it identifies the correct region with significant probability, another fault condition is seen to be identified with higher probability. Similar is the result if sensor $T_{24}$ is used as seen in Fig. 3(a) and Fig. 3(b). To resolve this ambiguity, the sensor information fusion framework makes use of both $P s_{30}$ and $T_{24}$ to correctly identify the fault in the parameter space without any ambiguity, as seen in Fig. 4(a) and Fig. 4(b). The estimates lie in the ranges ( $\pm 3 \sigma$ bound) of 0.9747 to 0.9753 for $\psi_{H P T}$ and $\zeta_{H P T}$ and 0.9847 to 0.9853 for $\psi_{L P T}$ and $\zeta_{L P T}$, respectively; in this case, highest probability is assigned to the training grid point that is nearest to the test point.

\section{Summary, CONClusions AND FutURE WORK}

This paper presents a symbolic dynamic filtering (SDF)based methodology for multiple-fault estimation in aircraft Gas turbine engines in a sensor-information-fusion framework. While there are many other issues that need to be addressed before the proposed estimation method can be 


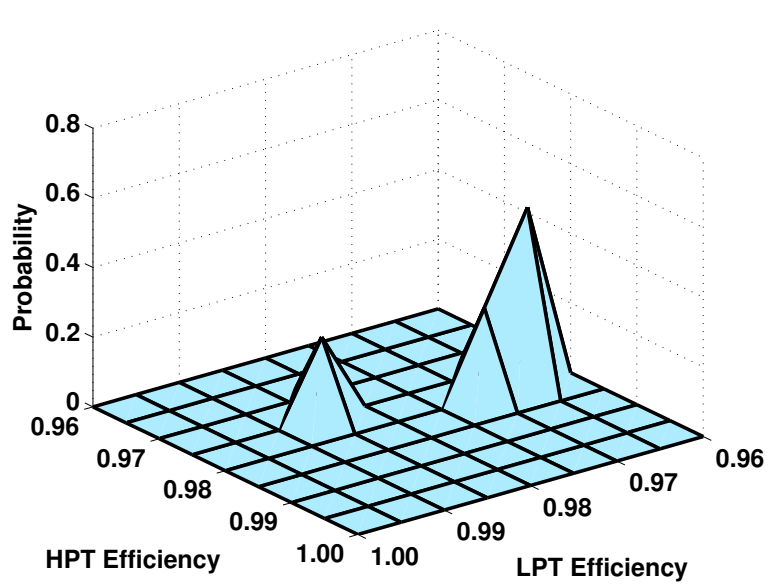

(a) Surface plot of fault estimation

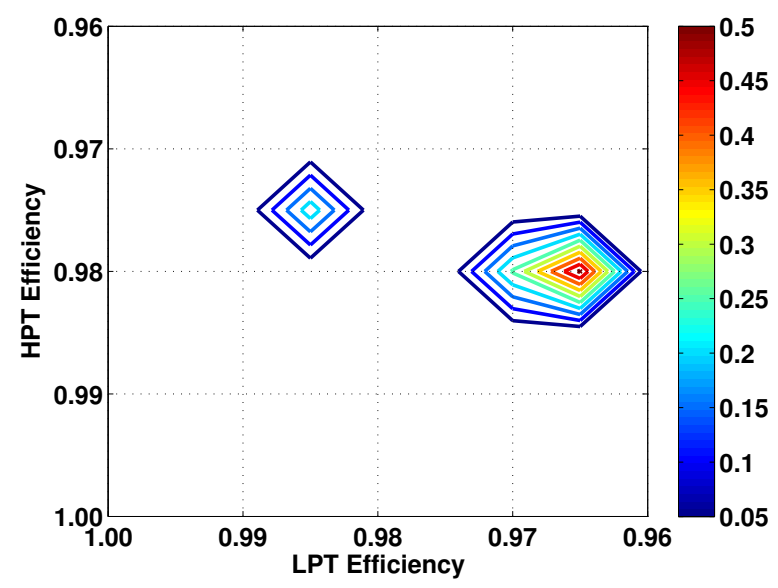

(b) Contour plot of fault estimation

Fig. 3. Fault estimation in HPT-LPT based on $T_{24}$ sensor

considered for real-life applications in commercial aircraft, the following research topics are being currently pursued.

- Identification of the mode (e.g., gradual deterioration, intermittently occurring, and abrupt large) of multiple fault evolution.

- Extension of Fault Estimation to Fault Prognosis by using usual deterioration profiles of the components with respect to cycles of operation.

- Optimal sensor selection based on the estimation results of the whole parameter space.

\section{REFERENCES}

[1] A. Hoffman and N. van der Merwe, "The application of neural networks to vibrational diagnostics for multiple fault conditions," Comput. Stand. Interfaces, vol. 24, no. 2, pp. 139-149, 2002.
[2] V. Boppana, I. Hartanto, and W. K. Fuchs, "Fault diagnosis using state information," in FTCS '96: Proceedings of the The Twenty-Sixth Annual International Symposium on Fault-Tolerant Computing (FTCS '96), (Washington, DC, USA), p. 96, IEEE Computer Society, 1996.

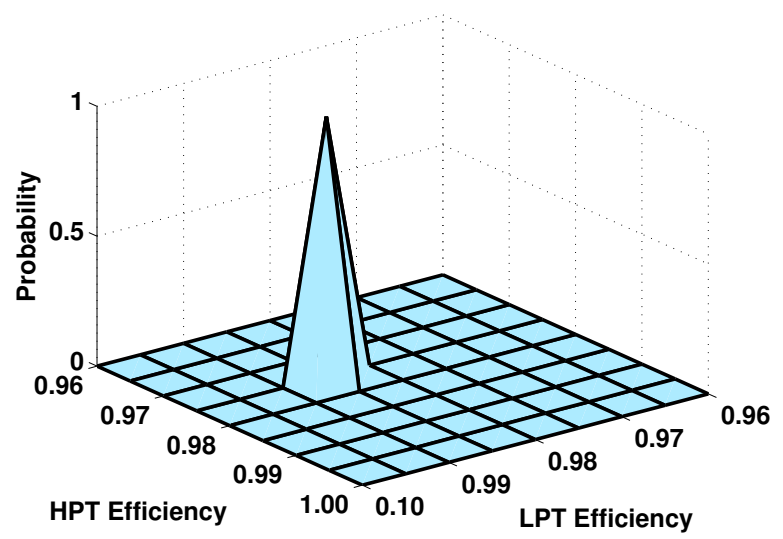

(a) Surface plot of fault estimation

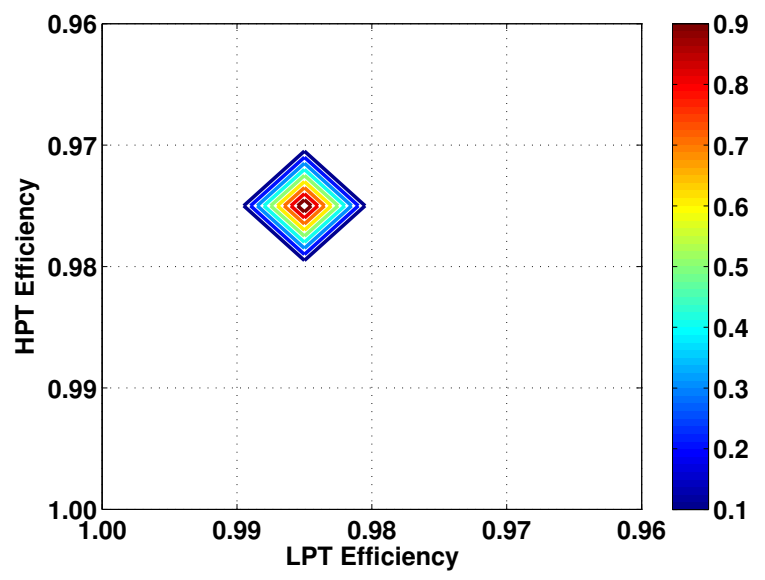

(b) Contour plot of fault estimation

Fig. 4. Fault estimation in HPT-LPT based on $P s_{30}$ and $T_{24}$ sensors

[3] S. Gupta, A. Ray, S. Sarkar, and M. Yasar, "Fault detection and isolation in aircraft gas turbine engines: Part i - underlying concept," Proceedings of the I Mech E Part G: Journal of Aerospace Engineering, vol. 222, no. 3, pp. 307-318, May 2008.

[4] S. Sarkar, M. Yasar, S. Gupta, A. Ray, and K. Mukherjee, "Fault detection and isolation in aircraft gas turbine engines: Part ii - validation on a simulation test bed," Proceedings of the I Mech E Part G: Journal of Aerospace Engineering, vol. 222, no. 3, pp. 319-330, May 2008.

[5] A. Ray, "Symbolic dynamic analysis of complex systems for anomaly detection," Signal Processing, vol. 84, no. 7, pp. 1115-1130, 2004.

[6] A. Subbu and A. Ray, "Space partitioning via hilbert transform for symbolic time series analysis," Applied Physics Letters, vol. 92, no. 8, pp. 084107-1 to 084107-3, February 2008.

[7] R. Duda, P. Hart, and D. Stork, Pattern Classification. John Wiley, New York, 2001.

[8] D. K. Frederick, A. DeCastro Jonathan, and J. S. Litt, "Users guide for the commercial modular aero-propulsion system simulation (c-mapss)," October 2007. NASA/TM2007-215026. 